This item was submitted to Loughborough's Research Repository by the author.

Items in Figshare are protected by copyright, with all rights reserved, unless otherwise indicated.

\title{
Contact force estimation in the wheel/rail interface for curving scenarios through regions of reduced adhesion
}

PLEASE CITE THE PUBLISHED VERSION

https://doi.org/10.1016/j.mechatronics.2017.06.013

\section{PUBLISHER}

(C) Elsevier

VERSION

AM (Accepted Manuscript)

\section{PUBLISHER STATEMENT}

This work is made available according to the conditions of the Creative Commons Attribution-NonCommercialNoDerivatives 4.0 International (CC BY-NC-ND 4.0) licence. Full details of this licence are available at: https://creativecommons.org/licenses/by-nc-nd/4.0/

\section{LICENCE}

CC BY-NC-ND 4.0

\section{REPOSITORY RECORD}

Hubbard, Peter D., Nabilah Farhat, Christopher P. Ward, and G.A. Amarantidis. 2019. "Contact Force Estimation in the Wheel/rail Interface for Curving Scenarios Through Regions of Reduced Adhesion". figshare. https://hdl.handle.net/2134/27371. 


\title{
Contact Force Estimation in the Wheel/Rail Interface for Curving Scenarios Through Regions of Reduced Adhesion
}

\author{
P.D.Hubbard ${ }^{\mathrm{a}, *}$, N.Farhat ${ }^{\mathrm{a}}$, C.P.Ward ${ }^{\mathrm{a}}$, G.A.Amarantidis ${ }^{\mathrm{a}}$ \\ ${ }^{a}$ Wolfson School of Mechanical, Electronic and Manufacturing Engineering, Sir David \\ Davies Building, Loughborough University, Loughborough, LE12 5JS
}

\begin{abstract}
Regions of extreme low-adhesion between the wheel and rail can cause critical problems in traction and braking. This can manifest in operational issues such as signals being passed at danger, or pessimistic network wide responses to mitigate for localised issues. Poor traction conditions can be caused by oil contaminants, rain, ice, condensation of water droplets (micro-wetting) or leaves on the line, where compressed leaf contamination can cause a rapid decrease in adhesion. The complexity of the problem arises as a result of the inability to directly measure and monitor all the factors involved. There remains a lack of real-time information regarding the state and location of low-adhesion areas across rail networks. On-board low adhesion detection technology installed to in-service vehicles is a suggested method to capture up-to-date adhesion information network wide and minimise significant disruptions and cancellations in railway schedules. This paper extends a principle of a model-based estimation technique previously developed in straight track running for operating in a curving scenario. The vehicle model of focus here will be a high-fidelity, multi-body physics representation of a full-vehicle.
\end{abstract}

Keywords: Rail, Low Adhesion, Wheel/Rail Contact, Kalman Filtering 2016 MSC: 00-01, 99-00

\footnotetext{
* Corresponding author

Email address: p.d.hubbard3@lboro.ac.uk (P.D.Hubbard)
}

Preprint submitted to Journal of Mechatronics

May 26, 2017 


\section{Introduction}

The development of railway systems present an exciting opportunity to mechatronic systems engineers. Unlike aerospace and automotive industry the uptake of advanced technology in design, such as including disruptive methods

5 [1] or intelligent condition monitoring [2], is relatively low and the basic function and layout of a railway suspension system is similar to that designed in the nineteenth century [3].

Real-time condition monitoring is a relatively new concept adopted by the rail industry. Available commercial systems such as 'ORBITA' by Bombardier [4] and Alstom's 'Traintracer' [5] have been released and largely identify faults by collecting signals over time and using trend analysis coupled with expert judgement to identify faults. Embedding intelligence and modern fault identification methods into a bogie provides an opportunity to increase the effectiveness of such systems. This is a natural additional inclusion of a mechatronic solution as the fundamental function needs of high-fidelity sensing and computing resources are inherent to design. Overall, Mechatronic solutions offer a more intelligent, efficient solution than the passive solutions are able to provide.

Although a fully mechatronic, intelligent system is some years away, ideas and technology that form a subset of this knowledge-base are available to be applied to in-service systems. A strong area of research is the use of model-based estimators to identify system parameters in real time. A number of publications such as [2], [6] and 7] present overviews of opportunities of available methods to the rail domain such as estimated the current parameter of suspension components. Use of these methods can better inform the overall health assessment of the asset and enable a transition from schedule-based to condition-based maintenance.

One opportunity of model-based estimation is to identify the adhesion level experience by a rail vehicle during normal running. Rail networks are at risk of being subject to areas of low adhesion. These adverse conditions can be generated by a number of environmental conditions that can be difficult to 
predict. Contaminants such as oil, rain, ice, condensation or compressed leaves can all contribute to low adhesion. Low adhesion can cause critical problems in traction and braking that can manifest in operational issues such as signals being passed at danger, or pessimistic network wide responses to mitigate for localised issues.

A previous study of low adhesion at micro-slip [8] suggested that for low adhesion conditions the initial gradient of the creep slope and the overall saturation level is lower for reduced coefficients of friction. Preceding this study, [9] showed that for a simple vehicle model, it was possible to estimate the contact forces experienced at the wheel/rail interface. The reduction in the initial gradient of the creep slope due to lower adhesion manifests as different vehicle dynamics to those experienced in good running conditions. The method presented by [9] uses the difference in these measured dynamic quantities to estimate the contact forces in the wheel/rail interface.

45

Further studies, 9], have gradually included increasingly realistic test scenarios by extending the simulation model to a half vehicle with non-linear contact mechanics [10], to industry standard high-fidelity multi-body physics models 11]. Each time, it has been shown that a good estimation of contact force is attainable across adhesion profiles using only a reduced order vehicle suspension model.

Recently, the study has been extended to investigate the method of contact force estimation during curving [12]. This technique used a reduced-order, linear suspension model of a simplified vehicle, and non-linear contact mechanics to simulate the vehicle travelling through a curve, and a reduced-order suspension model to estimate contact force.

The overall use of contact force estimation is to apply appropriate postprocessing to the results in order to infer the adhesion coefficient at the wheel/rail interface, as was investigated in [11]. A significant pre-cursor to this is first be able to identify contact force.

Enabling in-service vehicles to monitor and communicate adhesion levels across the network would build up a knowledge-base and enable the identifica- 
tion of at-risk areas in order to predict problems. This could inform mitigation strategies to target specific areas to reduce the impact on national Autumn time-tabling (which is often conservative) caused by low-adhesion events.

This paper shows the efficacy of contact force estimation during a curve. The method is applied to a high-fidelity, realistic, simulation of a rail vehicle to extend the study of [12]. A novel inclusion in this paper is the use of systems identification to approximate a linear, primary suspension model for use in a Kalman Filter.

\section{Method}

The method used to estimate contact force used by [9] uses an extended Kalman-Bucy filter 13] to evaluate sensor readings taken from a rail vehicle.

This research requires the generation of two distinct system models. As a real, in-service vehicle is not used in this study, a realistic simulation model is required. Such a model requires inclusion of non-linearities in both suspension movement and wheel/rail contact mechanics. The simulation should also allow definable curving characteristics to generate a range of test data. The simulation developed is discussed in Section 3 .

In addition, the Kalman-Bucy filter requires a reduced-order linear model to facilitate estimation. This model is designed to represent the lateral and yaw motions of the principle suspension components as this has been shown to adequately capture the key stability characteristics of a vehicle [3]. It will be required to show that the linear, reduced-order model sufficiently captures the system dynamics experienced by the simulation. An in-effective linear model will reduce the quality of the contact force estimations. The development and validation of this linear model is presented in section 4 .

The estimator is tested by providing sensor data from the simulation to the contact force estimator for a series of known adhesion conditions and curve profiles. The estimated contact forces is then be compared to those experienced in simulation and an evaluation of performance is made in section 5 


\section{Simulation Model}

Simpack 14] is a multi-body physics modelling package and is used across different engineering disciplines to provide high-fidelity simulations of mechanical systems. This piece of software allows complex and cross-coupled non-linearities to be included in the system to accurately represent real-world systems.

In this case, a full-vehicle model has been implemented in Simpack. Figure 1 provides an overview of the simulation model. The vehicle consists of a main body, two bogies and four wheelsets, each of which are modelled as 'masses' and free to move in six degrees of freedom. The suspension arrangement is typical of a modern vehicle with secondary air-bag suspension, secondary lateral dampers and a radius arm primary suspension. Suspension forces are caculated based on the relative displacement and velocities of the interacting bodies and include non-linearities such as bump-stops, variations in damping ratios, dead-bands, etc. to fully represent a real vehicle. The contact mechanics detailing the wheel/rail interaction are discussed below.

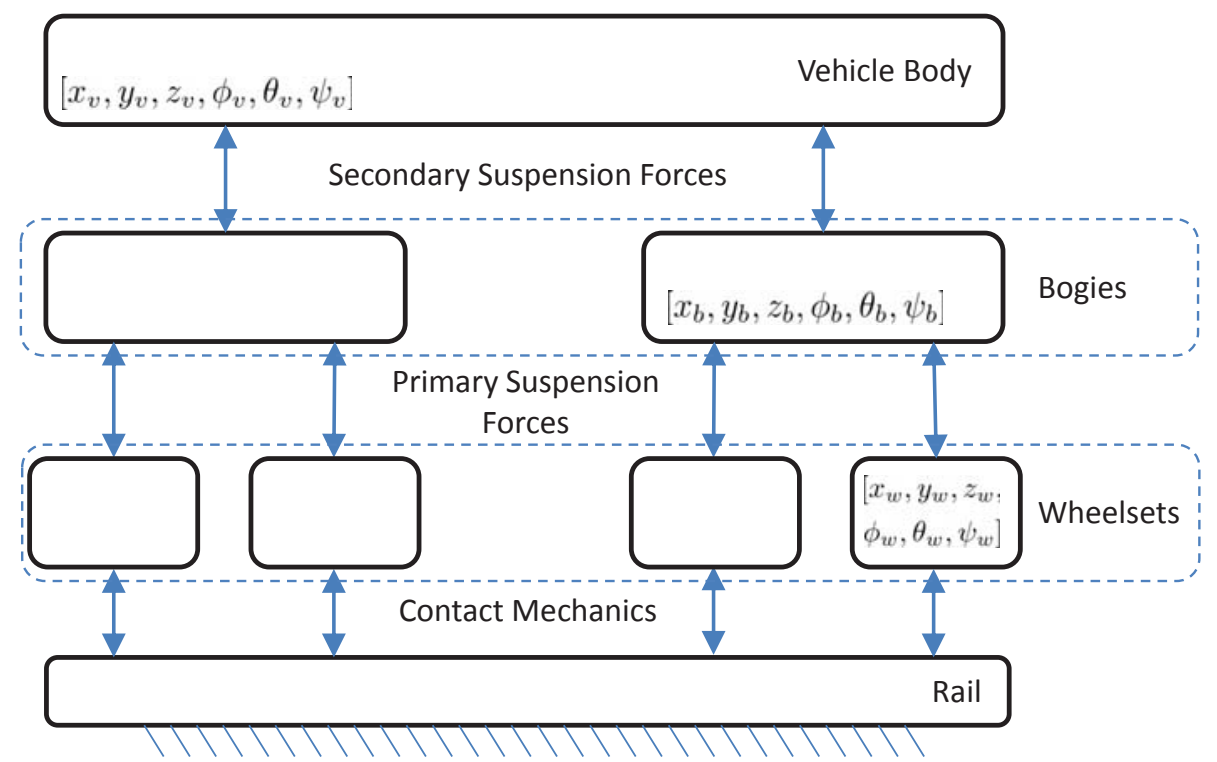

Figure 1: Overview of Multi-body physics model: Side view 
The vehicle model generated is based on a high speed passenger vehicle and has been used in previous studies such as [15]. The main principle parameters used to define the vehicle are found in Table 1.

\begin{tabular}{c|c|c} 
Name & Value & Units \\
\hline \hline Vehicle body mass & 20000 & $\mathrm{~kg}$ \\
Secondary yaw damper/actuator semi-spacing & 1.4 & $\mathrm{~m}$ \\
Bogie semi-wheelbase & 1.28 & $\mathrm{~m}$ \\
Bogie yaw inertia & 987 & $\mathrm{kgm}$ \\
Radius arm length & 0.45 & $\mathrm{~m}$ \\
Wheelset mass ( $\left.m_{w}\right)$ & 1200 & $\mathrm{~kg}$ \\
Wheelset yaw inertia $\left(I_{w x}\right)$ & 730 & $\mathrm{kgm}$ \\
Wheelset pitch inertia & 30 & $\mathrm{kgm}$ \\
Wheel rolling radius & 0.46 & $\mathrm{~m}$ \\
Half gauge width & 0.75 & $\mathrm{~m}$ \\
Load per wheelset & 78270 & $\mathrm{~N}$ \\
Primary shear spring lateral stiffness & $6.5 \times 10^{6}$ & $\mathrm{~N} / \mathrm{m}$ \\
Primary shear spring longitudinal stiffness & $3.14 \times 10^{6}$ & $\mathrm{~N} / \mathrm{m}$ \\
Axlebox lateral semi spacing & 1 & $\mathrm{~m}$ \\
Bush longitudinal stiffness & $12.2 \times 10^{6}$ & $\mathrm{~N} / \mathrm{m}$ \\
Bush lateral stiffness & $6 \times 10^{6}$ & $\mathrm{~N} / \mathrm{m}$ \\
Secondary yaw stiffness & $1.6 \times 10^{5}$ & $\mathrm{~N} / \mathrm{m}$ \\
Secondary shear spring lateral stiffness & $3.75 \times 10^{3}$ & $\mathrm{Nm} / \mathrm{rad}$ \\
Vehicle speed (V) & $250 \times 10^{3}$ & $\mathrm{~N} \mathrm{s/m}$ \\
\hline
\end{tabular}

Table 1: Nominal principle parameters for simulation model

\subsection{Wheel/Rail Interaction}

An important aspect of the simulation model is the way in which the wheel/rail interaction is addressed. It has been shown [16] that at low adhesion conditions appropriate non-linearities of contact are required to be included in the model to 
accurately represent system behaviour. The vehicle model uses a S1002 profile wheel, and a UIC60 railhead. From these profiles, the non-linear relationship of contact angle and rolling radii are calculated during the simulation. The simulation also includes track irregularities (i.e. lateral and vertical position, twist, variation in gauge width and cross level) typical of high-speed lines.

The creep forces due to creep are approximated using the Kalker Fastsim approach 17], and allows for the changing contact patch due to the movement of wheel to rail. This method also allows an adjustment of creep force to be made dependant on the coefficient of adhesion $(\mu)$ present. In this simulation, four different adhesion levels are selected that represent distinct operating conditions of rail vehicles. These are characterised in Table2 The condition where $\mu=0.06$ represents a dangerous operating condition where defensive driving is 125 required to minimise risks, and $\mu=0.03$ is an extreme condition where even adjusting driving style may not be sufficient for safety.

\begin{tabular}{c|c}
$\mu$ & Operating condition \\
\hline \hline 0.56 & Dry track \\
0.32 & Wet track \\
0.06 & Low adhesion \\
0.03 & Very low adhesion \\
\hline
\end{tabular}

Table 2: Characterisation of adhesion coefficient to operating condition

\subsection{Simulation Model data}

The vehicle in this simulation will be subject to a curving scenarios defined by the superelevation (cant angle) $\theta_{c}$ and the curve radius $R$. The vehicle will undergo a steady transition from straight track running into the steady state curve with the profile shown in Figure $2, R$ follows the same profile and transitions from 0 to $1168 \mathrm{~m}$.

Simpack has the capacity to record and output a large number of dynamic variables within the system. The key variables of interest in this investigation are those that capture the wheelset and bogie dynamics. Figures 3 and 4 show 


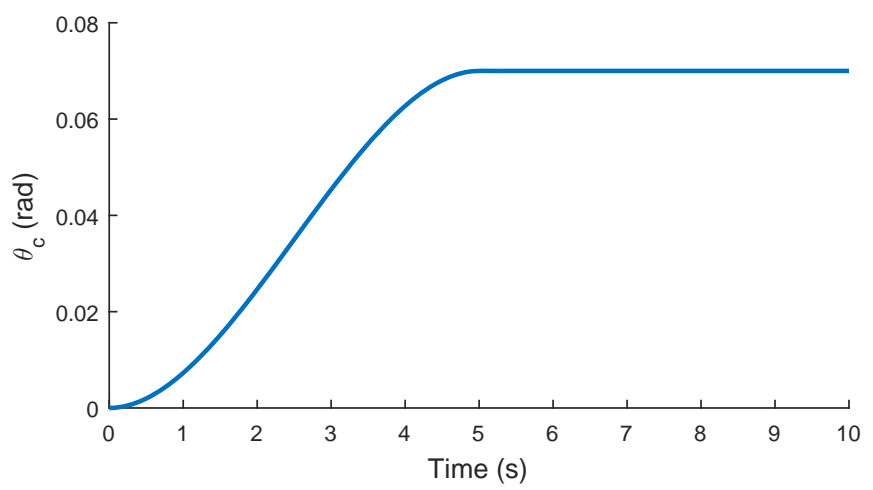

Figure 2: Transition of superelevation angle from straight track to curve at $40 \mathrm{~m} / \mathrm{s}$

the recorded wheelset lateral position and wheelset yaw position as a result of the simulation of the transition into the curve at different adhesion conditions.

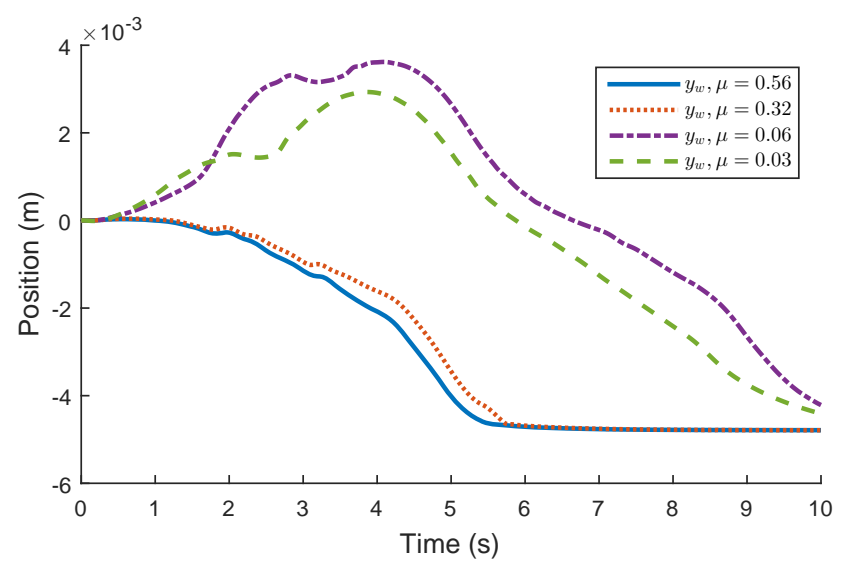

Figure 3: Simulation of curve transit - wheelset lateral position

It can be seen that at lower levels of adhesion, the wheelset struggles to align itself to the steady state curving condition. This is anticipated as the contact forces are much smaller and are unable to easily overcome the straightening action of the suspension components.

The high-quality simulation model developed here is a beneficial intermediary stage between simple linear models and real system testing for new tech- 


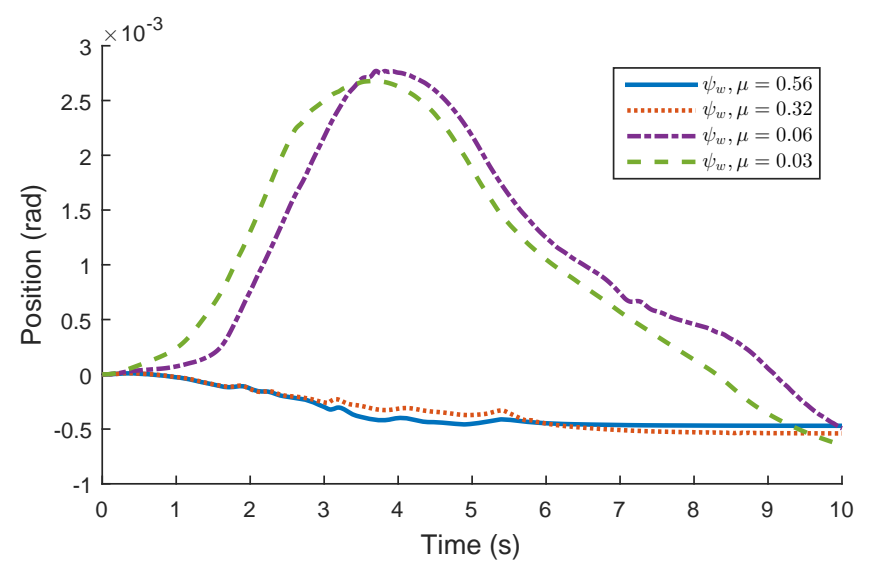

Figure 4: Simulation of curve transit - wheelset yaw position

nology such as this contact force estimation method. The main advantage is that the simulation will output creep forces experienced by the rail vehicle and therefore provide a frame of reference that is not available on real vehicles. The variables captured from the simulation will be treated and analysed as if they were data captured from sensors on-board and a further benefit of simulation is that there is no real limit to the amount of data that can be captured. This therefore provides useful information on specifying sensors for in-service testing. At this stage in development it is useful to assume that necessary variables are directly measurable.

These four test runs will provide the data required for generating the linear suspension model, as described in section 4.2 .

\section{Kalman Filter Development for C.F. Estimation}

This approach uses an extended Kalman-Bucy method to estimate the contact forces between the wheel and the rail. This method was introduced by [18], and developed further using increasingly realistic simulations [9, 19, 11, 16]. These investigations showed that the estimator was able to effectively attain the total contact force, but unable to differentiate between creep forces and reactive normal forces due to contact and roll angles. The intention in this 
section is to adjust the formation of this filter in order to enable contact force estimation during curving transit.

To populate the Kalman filter, is is required to generate a linear model of the system that takes the standard state space form:

$$
\begin{gathered}
\dot{\mathbf{x}}=\mathbf{A}_{\mathbf{k}} \mathbf{x}+\mathbf{B}_{\mathbf{k}} \mathbf{u} \\
\mathbf{y}=\mathbf{C}_{\mathbf{k}} \mathbf{x}
\end{gathered}
$$

The full description of the chosen states and parameters in order to estimate adhesion will be presented in 4.1 .

The Kalman-Bucy filter calculates the updated estimates of the state $\dot{\hat{\mathbf{x}}}$ using both the state-space description and an adjustment factor from the difference in system measurements, as described by

$$
\begin{gathered}
\dot{\hat{\mathbf{x}}}=\mathbf{A}_{\mathbf{k}} \hat{\mathbf{x}}+\mathbf{B}_{\mathbf{k}} \hat{\mathbf{u}}+\mathbf{K}(\mathbf{y}-\hat{\mathbf{y}}) \\
\hat{\mathbf{y}}=\mathbf{C}_{\mathbf{k}} \hat{\mathbf{x}}
\end{gathered}
$$

The method to be demonstrated in this section is concerned with measuring a minimal set of vehicle running dynamics and estimating the contact force between the wheel and the rail. Simpack is used as a simulation tool to provide realistic running dynamics for prescribed operating conditions. The running dynamics are then assessed by the Kalman-Bucy filter to obtain estimates of the contact forces experienced between the wheel and the rail. As Simpack is a software simulation, a direct comparison between estimated and simulated contact forces can be made to assess the performance of the force estimation. As an aside, it is worth noting that this comparison would be some-what more difficult should an in-service vehicle be used as a test subject as this measurement cannot be made directly. The simulation method therefore provides a useful middle ground to assess the efficacy of the estimation method. 


\subsection{Linear Plan-View Model Development}

The model used to populate the estimator is founded in comparing the force balance equations of a single wheelset in the lateral and yaw directions of motion. This section describes how this model is constructed using systems identification.

Consider the coupled differential equation sets that describe the mechanical oscillation of a wheelset in yaw and lateral position $y_{w}$ and $\psi_{w}$

$$
\begin{gathered}
m_{w} \ddot{y}_{w}=F_{\text {creep }}+F_{g}+F_{\text {sus }}+F_{\text {curve }} \\
I_{w x} \ddot{\psi}_{w}=M_{\text {creep }}+M_{\text {sus }}
\end{gathered}
$$

where $F_{\text {creep }}$ describes the sum of the lateral creep forces $F_{y L}, F_{y R}$ on the wheelset. $M_{\text {creep }}$ is the resultant moment acting upon the wheelset due to the longitudinal contact forces on the left and right wheel $F_{x L}, F_{x R}$ at the moment arm $a$, and is given by:

$$
M_{\text {creep }}=a F_{x L}-a F_{x R}
$$

Other terms exist that could be included to describe $M_{\text {creep }}$ but it can been shown that these are negligible in comparison to the moment induced due to the longitudinal creep forces, even at lower levels of adhesion.

$F_{g}$ is the 'gravitational stiffness' (i.e. the resolved normal force due to the contact angles on the left and right wheel $\delta_{L}, \delta_{R}$ and differential rolling radii $\left.r_{L}-r_{R}\right)$ and is given by:

$$
F_{g}=W\left(\frac{\delta_{L}-\delta_{R}}{2}+\frac{r_{l}-r_{R}}{2 a}\right)
$$

As the vehicle is transitioning through a curve of radius $R$ and a cant angle of $\theta_{c}$, the wheelset is subject to centripetal force and a gravitational force due to cant angle. These are captured in the term $F_{\text {curve }}$ which is described by:

$$
F_{\text {curve }}=m_{w} g \sin \theta_{c}-m_{w} \frac{V^{2}}{R}
$$


The lateral suspension force $F_{\text {sus }}$ and the yaw suspension moment $M_{\text {sus }}$ are the force and moment created by the suspension deflection due to the differential position and speed of the wheelset with respect to the bogie. The force and moment are represented as generic functions of the wheelset and bogie dynamic quantities

$$
\begin{gathered}
F_{\text {sus }}=f_{s}\left(y_{w}, \dot{y}_{w}, \psi_{w}, \dot{\psi}_{w}, y_{b}, \dot{y}_{b}, \psi_{b}, \dot{\psi}_{b}\right) \\
M_{\text {sus }}=f_{m}\left(y_{w}, \dot{y}_{w}, \psi_{w}, \dot{\psi}_{w}, y_{b}, \dot{y}_{b}, \psi_{b}, \dot{\psi}_{b}\right)
\end{gathered}
$$

\subsection{Parameter Identification for Suspension Description}

In order to complete the linear description of the wheelset, an expression describing the linearised suspension system in yaw and lateral motion is required

In previous work [11, 16] this model has been produced from first principles (i.e. gathering the Newtonion and Euler equations that describe the system). In this case, systems identification will be used to obtain the linearised functions $f_{s}$ and $f_{m}$.

Using this data-driven method to obtain the model can have an advantage over the first principles methods. In low adhesion scenarios, the suspension deflection can be quite small and expose different operating modes to normal running. For example, the forces experienced may not be high enough to breakout suspension components from static friction[11]; thus negating the component for this scenario. This method relies on a linear model that is valid across a range of operating conditions.

The disadvantage of this method is that the model produced is tuned to the scenario from which the data is generated. Should the operating point change to an unfamiliar location, the identified model may not longer represent the current system dynamics.

The parameter identification method used creates a regression model formed from the least squares minimisation of the error between a measured output variable, and the estimated variable as a function of defined states [20]. 
The general form of the model generation is given by:

$$
\boldsymbol{\Theta}=\left(\mathbf{X}^{T} \mathbf{X}\right)^{-1} \mathbf{X}^{T} Y
$$

where $Y$ is the time history of the output variable, and $\mathbf{X}$ is the time history the state variables and can be later used in a state model.

The estimate of the output variable can then be formed through

$$
\hat{Y}=\mathbf{X} \Theta
$$

This section will describe how the simulation outputs will be used to provide the measured output of suspension forces, along with a time history of the required states (in the form of lateral and yaw dynamics) in order to identify coefficients of state variables that relate the two.

Consider (5) when re-arranged to make $F_{\text {sus }}$ the subject.

$$
F_{\text {sus }}=m_{w} \ddot{y}_{w}-F_{\text {creep }}-F_{g}-F_{\text {curve }}
$$

Using the variables output from the vehicle simulation, the terms on the right hand side of (14) are straightforward to ascertain using equations (8), (9). The difficulty lies in expressing $F_{\text {sus }}$ as it is a complex combination of terms 235 using simulation data and (14), a numerical solution can be obtained for the time history of $F_{\text {sus }}$ that was experienced during simulation.

Before parameter identification takes place, a simplification can be made to the state variables. The suspension force is due to the relative position and velocity of the wheelset to bogie. In terms of the stiffnesses and damping due to only lateral movement, the variables of interest are not the absolute positions and velocity of the two bodies, but the relative motions of the two. This is not true for other terms as the geometry of the suspension requires that they are treated independently. To approximate $F_{s} u s$, the state variables are defined to 


$$
\hat{F}_{\text {sus }}=\left[\begin{array}{llllll}
\left(y_{w}-y_{b}\right) & \left(\dot{y}_{w}-\dot{y}_{b}\right) & \psi_{w} & \dot{\psi}_{w} & \psi_{b} & \dot{\psi}_{b}
\end{array}\right]^{T} \Theta_{F}
$$

This now provides an expression for $F_{\text {sus }}$ in terms of the variables expressed in (10). Using the parameter identification method in (12), and a time history of the required states from the simulation model, the resultant value of $\boldsymbol{\Theta}_{F}$ is shown in the application of equation (13):

$$
\hat{F}_{\text {sus }}=\mathbf{X}\left[\begin{array}{llllll}
1.318 & -0.085 & -2.253 & 0.837 & 0.620 & -0.854
\end{array}\right] \times 10^{7}
$$

As before, the right hand side of (17) can be generated directly from simulation data to provide a numerical solution for $M_{\text {sus }}$. In this case, the suspension geometry does not allow for any combination of terms so the state variables required to be defined for $M_{\text {sus }}$ are

$$
\hat{M}_{s u s}=\left[\begin{array}{llllllll}
y_{w} & \dot{y}_{w} & \psi_{w} & \dot{\psi}_{w} & y_{b} & \dot{y}_{b} & \psi_{b} & \dot{\psi}_{b}
\end{array}\right]^{T} \Theta_{M}
$$

Again, the minimisation of error is performed using (12), and the resultant value of $\boldsymbol{\Theta}_{M}$ is shown in the application of equation (13):

$$
\hat{F}_{\text {sus }}=\mathbf{X}\left[\begin{array}{llllllll}
-1.051 & -0.051 & 2.149 & 0.766 & 0.892 & 0.081 & -0.800 & -0.602
\end{array}\right] \times 10^{7}
$$




\subsection{Validation of linear model}

To directly evaluate the linear suspension model, a comparison can be made between $F_{\text {sus }}$ (which is directly extracted from the simulation) and $\hat{F}_{\text {sus }}$, which (he 'measured' position and velocities of the wheelset and bogie. A similar comparison can be made for the simulated and linearised yaw moment results.

Figures 5 and 6 show the simulated values compared to the linearised suspension model for curve transition at the highest and lowest adhesion levels 270 $(\mu=0.56$, and $\mu=0.03)$. Correlation metrics for simulations at all four adhesion levels can be found in Table 6. Correlation is assessed using the Sprague and Geers method [21]. This method assesses a 'goodness of fit' for magnitude and phase independently, with a combined result provided. A score of zero represents perfect correlation, and a score of one represents no correlation.

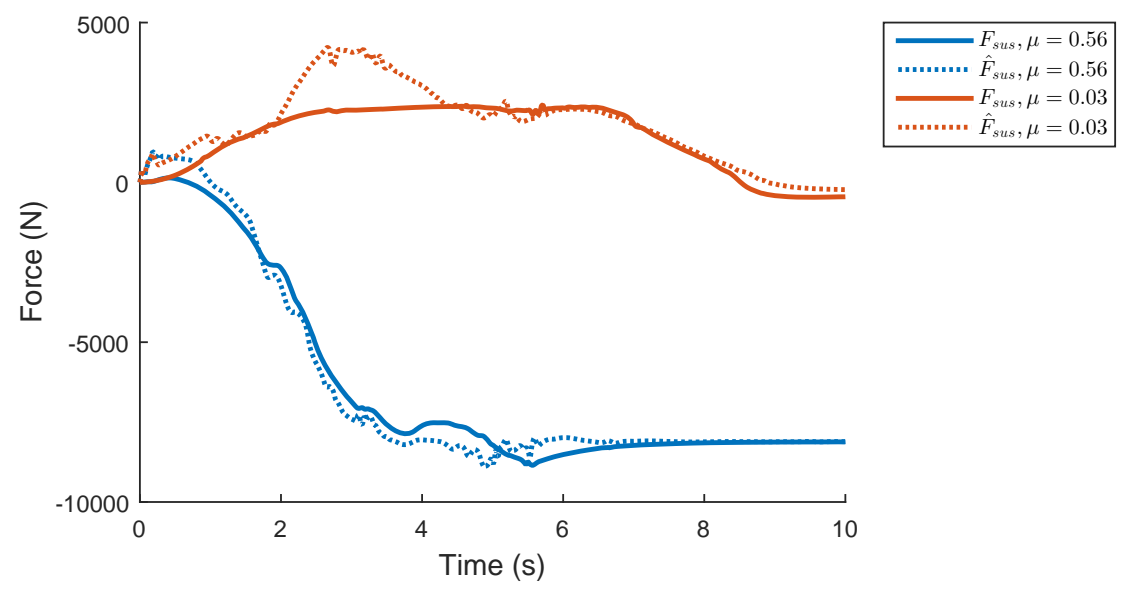

Figure 5: Simulated lateral suspension force vs linearised suspension force

Although correlation is good overall, It can be seen that this reduces with adhesion level. There are two anticipated reasons for this. Firstly the numerical values of contact force and moment are smaller at lower adhesion values so similar error sizes to those experienced at higher adhesion conditions relate to a larger percentage difference. Secondly, the suspension deflection at lower 


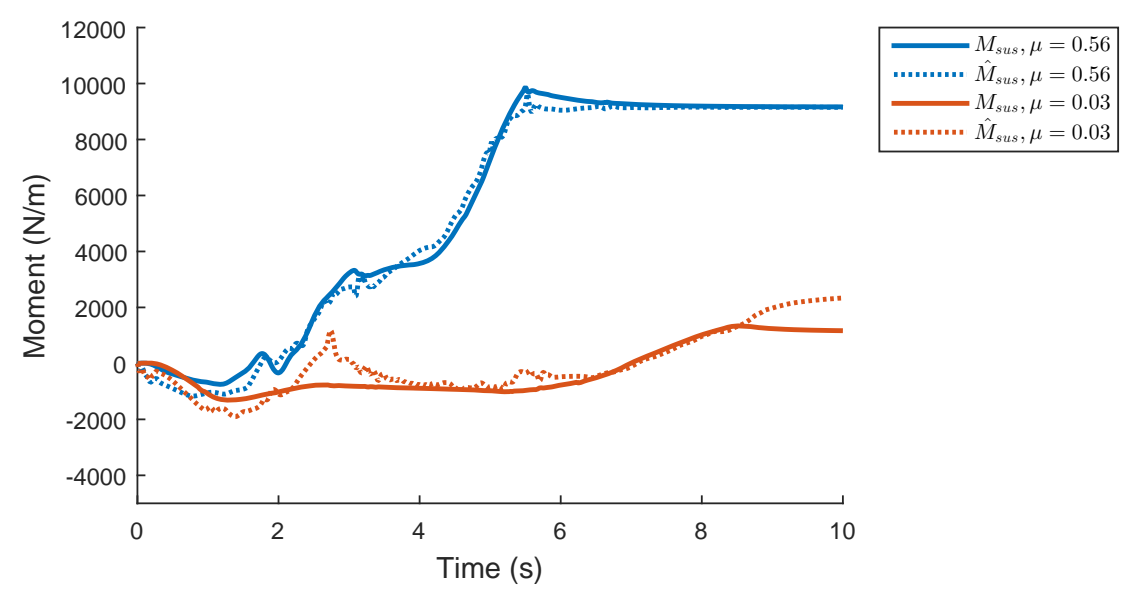

Figure 6: Simulated yaw suspension moment vs linearised suspension moment

\begin{tabular}{c|ccc|ccc} 
& \multicolumn{3}{|c|}{$\hat{F}_{\text {sus }}$ vs. $F_{\text {sus }}$} & \multicolumn{3}{c}{$\hat{M}_{\text {sus }}$ vs. $M_{\text {sus }}$} \\
\hline \hline$\mu$ & $\mathrm{M}$ & $\mathrm{P}$ & $\mathrm{C}$ & $\mathrm{M}$ & $\mathrm{P}$ & $\mathrm{C}$ \\
0.56 & 0.0098 & 0.0187 & 0.0211 & 0.0093 & 0.0153 & 0.0179 \\
0.32 & 0.0219 & 0.0310 & 0.0380 & 0.0138 & 0.0198 & 0.0241 \\
0.06 & 0.1500 & 0.0666 & 0.1641 & 0.0759 & 0.0797 & 0.1100 \\
0.03 & 0.2475 & 0.0904 & 0.2635 & 0.2048 & 0.1585 & 0.2589 \\
\hline
\end{tabular}

Table 3: linear suspension model correlation for M (magnitude), P (phase) and C (combined)

adhesions is smaller [11]. In these operating conditions more non-linearities within the suspension are exposed (such as breakout forces) and therefore a reduction in the quality of fit to a linear model is observed. At the most extreme operating condition of very low adhesion these effects are most pertinent. The yaw moment appears to be a better quality approximation overall, apart from at the lowest adhesion level. 


\subsection{State Model Definition}

Now that $F_{\text {sus }}$ and $M_{\text {sus }}$ have been described, the state space description outlined in Equations (11) and (2) can be completed. The states are defined as:

$$
\mathbf{x}=\left[\begin{array}{llllll}
y_{w} & \dot{y}_{w} & \psi_{w} & \dot{\psi}_{w} & F_{y F} & M_{\psi F}
\end{array}\right]^{T}
$$

where $F_{y F}$ and $M_{\psi F}$ are the total lateral force and total yaw moment due to contact that act on the wheelset and can be represented by:

$$
\begin{gathered}
F_{y F}=F_{\text {creep }}+F_{g} \\
M_{\psi F}=M_{\text {creep }}
\end{gathered}
$$

where minor terms have been neglected. They are included in the state vector as 'augmented states' as the full description of $\dot{F}_{\text {sus }}$ and $\dot{M}_{\text {sus }}$ is not present within $\mathbf{A}_{\mathbf{k}}$ and $\mathbf{B}_{\mathbf{k}}$.

The input vector $\mathbf{u}$ is defined as:

$$
\mathbf{u}=\left[\begin{array}{llllll}
y_{b} & \dot{y}_{b} & \psi_{b} & \dot{\psi}_{b} & \theta_{c} & R^{-1}
\end{array}\right]
$$

Although these are not controlled inputs to the system, they are defined as such to exclude them from being estimated within the Kalman filter.

The output vector $\mathbf{y}$ is defined such that the wheelset position and velocity (in both lateral and yaw directions) are output.

$$
\begin{aligned}
\mathbf{y} & =\left[\begin{array}{cccccc}
1 & 0 & 0 & 0 & 0 & 0 \\
0 & 1 & 0 & 0 & 0 & 0 \\
0 & 0 & 1 & 0 & 0 & 0 \\
0 & 0 & 0 & 1 & 0 & 0
\end{array}\right] \mathbf{x} \\
& =\left[\begin{array}{llll}
y_{w} & \dot{y}_{w} & \psi_{w} & \dot{\psi}_{w}
\end{array}\right]^{T}
\end{aligned}
$$


Using the results from the parameter identification, the matrices $\mathbf{A}_{\mathbf{k}}$ and $\mathbf{B}_{\mathbf{k}}$ are give as:

$$
\begin{aligned}
\mathbf{A}_{\mathbf{k}} & =\left[\begin{array}{cccccc}
0 & 1 & 0 & 0 & 0 & 0 \\
-790 & -310 & 20400 & -472 & \frac{1}{m_{w}} & 0 \\
0 & 0 & 0 & 1 & 0 & 0 \\
-1920 & 272 & -3201 & -147 & 0 & \frac{1}{I_{w x}} \\
0 & 0 & 0 & 0 & 0 & 0 \\
0 & 0 & 0 & 0 & 0 & 0
\end{array}\right] \\
\mathbf{B}_{\mathbf{k}} & =\left[\begin{array}{cccccc}
0 & 1 & 0 & 0 & 0 & 0 \\
790 & 310 & -23400 & 647 & g & -V^{2} \\
-1282 & -372 & 8620 & -407 & 0 & 0 \\
0 & 0 & 0 & 0 & 0 & 0 \\
0 & 0 & 0 & 0 & 0 & 0
\end{array}\right]
\end{aligned}
$$

The state space description is arranged such that when applied to the KalmanBucy filter, the estimated states are updated based upon the mathematical equation of the force balance equation on the wheelset. This is then adjusted from the difference in systems output, i.e. the differences in estimated and measured wheelset position and velocity (in lateral and yaw motion), multiplied by the Kalman gain $\mathbf{K}$.

The Kalman gain is obtained by selecting weighting attributes that reflect the degree of certainty of each state variable. In order to estimate the augmented states $F_{\text {sus }}$ and $M_{\text {sus }}$, the weighting matrices are chosen to reflect the high certainty in the directly measured states (i.e. $y_{w}, \dot{y}_{w}, \psi_{w}, \dot{\psi}_{w}$ ) and the high uncertainty in the unmeasured (augmented) states. The values for the weighting matrices $\mathbf{Q}_{\mathbf{k}}$ and $\mathbf{R}_{\mathbf{k}}$ were found to give the best results when chosen to be:

$$
\mathbf{Q}_{\mathbf{k}}=\operatorname{diag}\left[\begin{array}{llllll}
1 \times 10^{-6} & 1 \times 10^{-3} & 1 \times 10^{-6} & 1 \times 10^{-3} & 1 \times 10^{12} & 1 \times 10^{12}
\end{array}\right]
$$




$$
\mathbf{R}_{\mathbf{k}}=\operatorname{diag}\left[\begin{array}{llll}
1 \times 10^{-6} & 1 \times 10^{-3} & 1 \times 10^{-6} & 1 \times 10^{-3}
\end{array}\right]
$$

The numerical solution for $K$ is found by solving the Riccati equation in order to minimise the cost function defined by the weighting matrices $\mathbf{Q}_{\mathbf{k}}$ and $\mathbf{R}_{\mathbf{k}}$, and is performed using the 'lqe' command in Matlab [22].

The contact forces are then obtained from interrogating the estimated state vector $\hat{\mathbf{x}}$ following a simulation inclusive of the Kalman-Bucy filter.

\section{Contact Force Estimation during Curve Transit}

The test vehicle simulation was subject to a different curving scenario to that used to obtain the linear suspension model using parameter identification, as well as the original curve profile. The two curve profiles chosen are summarised in Table 4. Each curve is designed to provide approximately a 10 percent cant deficiency.

\begin{tabular}{c|cc} 
Curve & $\mathrm{R}(\mathrm{m})$ & $\theta_{c}(\mathrm{rad})$ \\
\hline \hline 1 & 1168 & 0.0700 \\
2 & 920 & 0.0774 \\
\hline
\end{tabular}

Table 4: Curving conditions

The transitions to these values occur over the same timebase as those high325 lighted in Figure 2 For each curve, the simulation was repeated for the four different adhesion levels $(\mu)$ as described in Table 2, Following the simulation, the dynamic quantities required were assessed using the Kalman-Bucy filter and the estimated lateral contact force $\hat{F}_{y F}$ and estimated yaw moment due to contact $\hat{M}_{y F}$ were extracted from the time history of the estimated state vector. The estimation results for curve 1 are presented in Figures 7 and 8 , and the estimation results for curve 2 are found in Figures 7 and 8 . The Sprague and Geers correlation metric for each adhesion scenario of curve 1 is found in Table 6. and those for curve 2 are found in Table 6 . 


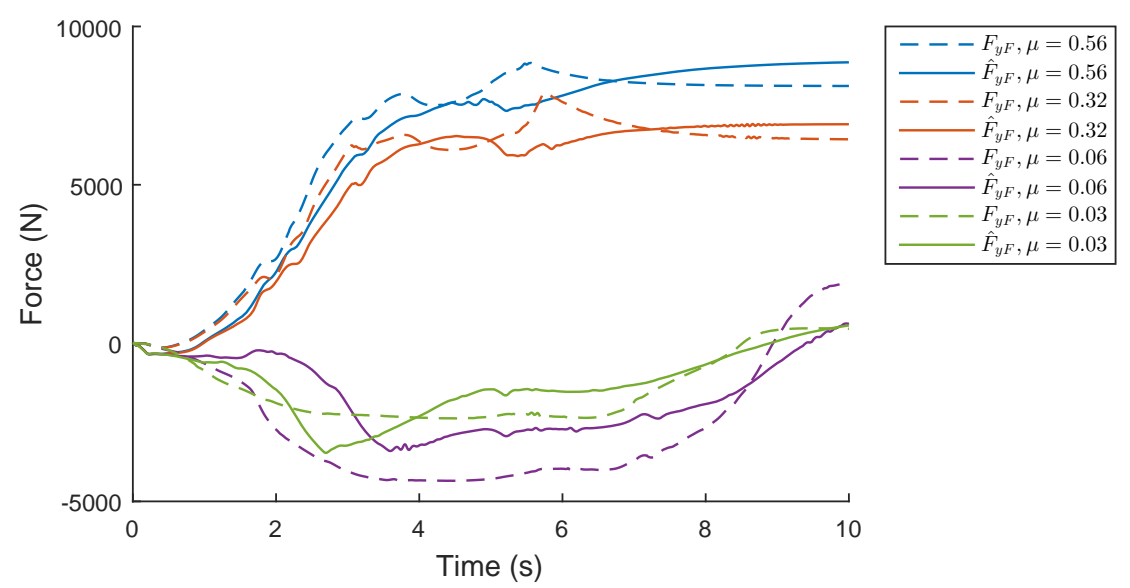

Figure 7: Curve 1 - Estimated and simulated lateral contact force $(R=1168)$

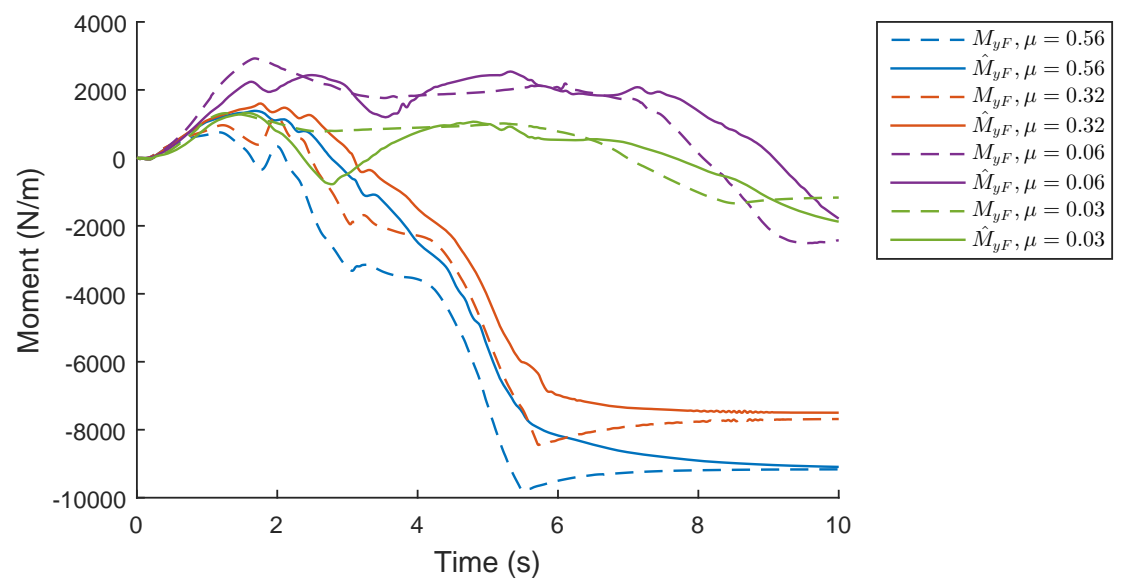

Figure 8: Curve 1 - Estimated and simulated yaw moment due to contact $(R=1168)$

\section{Discussion}

It is apparent from the results that contact moment is more accurately approximated than lateral creep force. This is not only seen by the time response of the different scenarios, but confirmed by the correlation metrics. Low worst numerical results appear at the low adhesion conditions. These scenarios provide challenging estimation conditions due to the smaller suspension deflections 


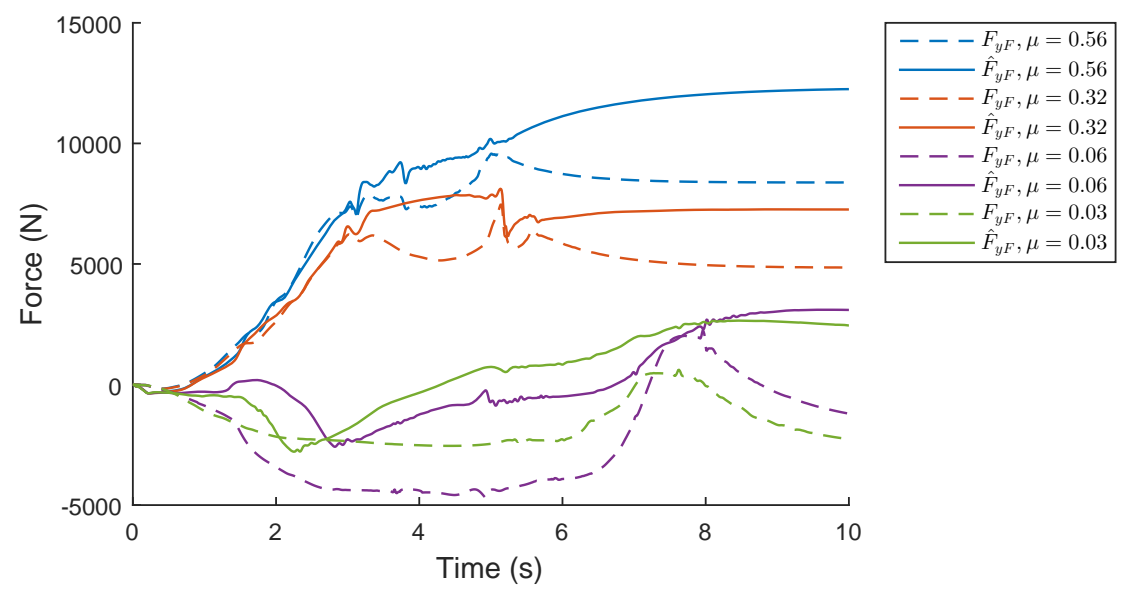

Figure 9: Curve 2 - Estimated and simulated lateral contact force $(R=900)$

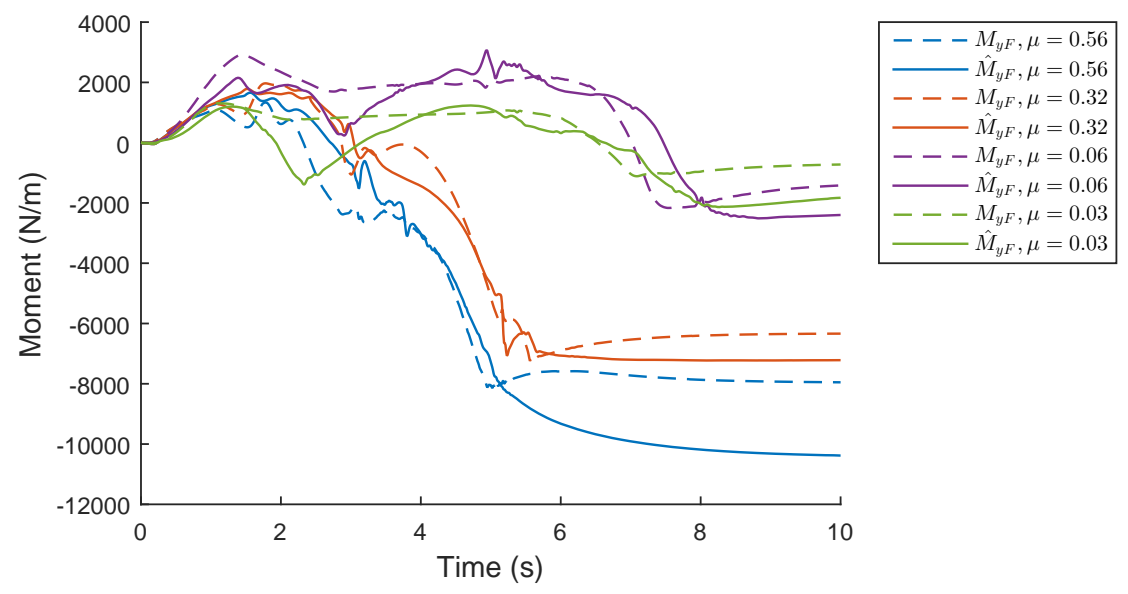

Figure 10: Curve 2 - Estimated and simulated yaw moment due to contact $(R=900)$

introduced. These findings are similar in many ways to the straight track simulation testing conducted previously [11].

It is clear from the results that the system is operating in different characteristic ways for smaller $\mu$ than in higher adhesion scenarios. It could be argued that if the desire was to provide accuracy at low adhesion levels, then 345

the suspension model could be identified at these conditions. This would sac- 


\begin{tabular}{c|ccc|ccc} 
& \multicolumn{3}{|c|}{$\hat{F}_{y F}$ vs. $F_{y F}$} & \multicolumn{3}{c}{$\hat{M}_{\psi F}$ vs. $M_{\psi F}$} \\
\hline \hline$\mu$ & $\mathrm{M}$ & $\mathrm{P}$ & $\mathrm{C}$ & $\mathrm{M}$ & $\mathrm{P}$ & $\mathrm{C}$ \\
0.56 & 0.0200 & 0.0315 & 0.0374 & 0.1006 & 0.0532 & 0.1137 \\
0.32 & 0.0358 & 0.0340 & 0.0494 & 0.0958 & 0.0448 & 0.1057 \\
0.06 & 0.5621 & 0.0928 & 0.5697 & 0.0821 & 0.1474 & 0.1687 \\
0.03 & 0.0748 & 0.1047 & 0.1287 & 0.1172 & 0.1941 & 0.2267 \\
\hline
\end{tabular}

Table 5: Curve 1 - Contact Force Estimation Correlation for M (magnitude), P (phase) and C (combined)

\begin{tabular}{c|ccc|ccc} 
& \multicolumn{3}{|c|}{$\hat{F}_{y F}$ vs. $F_{y F}$} & \multicolumn{3}{c}{$\hat{M}_{\psi F}$ vs. $M_{\psi F}$} \\
\hline \hline$\mu$ & $\mathrm{M}$ & $\mathrm{P}$ & $\mathrm{C}$ & $\mathrm{M}$ & $\mathrm{P}$ & $\mathrm{C}$ \\
0.56 & -0.2237 & 0.0405 & 0.2273 & -0.1804 & 0.0460 & 0.1862 \\
0.32 & -0.2386 & 0.0422 & 0.2423 & -0.0841 & 0.0306 & 0.0895 \\
0.06 & 0.8172 & 0.3574 & 0.8920 & 0.0157 & 0.1383 & 0.1392 \\
0.03 & 0.0828 & 0.5148 & 0.5214 & -0.2575 & 0.2485 & 0.3579 \\
\hline
\end{tabular}

Table 6: Curve 2 - Contact Force Estimation Correlation for M (magnitude), P (phase) and C (combined)

rifice performance at high adhesion conditions but could still be operationally beneficial, for example if distinguishing between 'low' and 'very low' conditions was important.

It can also be seen from the results that the overall level of yaw moment due to contact is reduced at lower conditions, and reasonably estimated to be lower at these conditions. It seems reasonable that some inference could be made about the adhesion coefficient using yaw moment in curving as an indicator.

\section{Conclusion}

The findings from this paper are positive in that an estimation of contact 355 formce through a curve is attainable. To refine and validate this technique, a further variety of testing scenarios (curve profile variations, adhesion levels, 
vehicle types, etc) as well as studies into sensor optimisation are required prior to vehicle testing.

In addition, a study is required into suitable post-processing techniques to provide operationally relevant data such as adhesion level, or wear characteristics of certain vehicles. Adhesion level post-processing has been addressed in [19], but not during curving.

Finally, a systems level study is required to identify the best method of capturing and distributing relevant data (or inferred information) to appropriate stakeholders in order to inform network wide mitigation strategies in servicing and timetabling.

\section{References}

[1] C. P. Ward, M. Jani, S. Dunnett, Mechatronic bogies: what are the benefits of adoption and how do we re European Railway Review.

URL http://www. europeanrailwayreview.com/23456/supplements/bogies-wheelsets-supplement/

[2] C. P. Ward, P. F. Weston, E. J. C. Stewart, H. Li, R. M. Goodall, C. Roberts, T. X. Mei, G. Charles, R. Dixon, Condition monitoring opportunities using vehicle-based sensors, Proceedings of the Institution of Mechanical Engineers, Part F: Journal of Rail and Rapid Transit 225 (2) (2011) 202-218.

[3] A. H. Wickens, The dynamics of railway vehiclesfrom Stephenson to Carter, Proceedings of the Institution of Mechanical Engineers, Part F: Journal of Rail and Rapid Transit 212 (3) (1998) 209-217. doi:10.1243/0954409981530805.

[4] Bombadier, Innovation \& Technology: ORBITA predictive asset management, the future of fleet mainte (2016).

URL http://www . bombardier.com/en/transportation/productsservices/services/innovation-te

[5] Alstom, Trainlife Service: Flexibility \& Partnership (2016).

URL http://www . alstom.com/Global/US/Resources/Documents/DownloadCentre/Brochure-TrainLi 
[6] P. Li, R. Goodall, P. Weston, C. Seng Ling, C. Goodman, C. Roberts, Estimation of railway vehicle suspension parameters for condition monitoring,

Control Engineering Practice $15 \quad$ (1) (2007) 43-55.

doi:http://dx.doi.org/10.1016/j.conengprac.2006.02.021.

URL http://www.sciencedirect.com/science/article/pii/S0967066106000463

390

[7] S. Bruni, R. Goodall, T. X. Mei, H. Tsunashima, Control and monitoring for railway vehicle dynamics, Vehicle System Dynamics 45 (7-8) (2007) 743-779. doi:10.1080/00423110701426690. URL http://www.tandfonline.com/doi/abs/10.1080/00423110701426690

[8] D. I. Fletcher, A new two-dimensional model of rolling-sliding contact creep curves for a range of lubrication types, Proceedings of the Institution of Mechanical Engineers, Part J: Journal of Engineering Tribologydoi:10.1177/1350650112465694.

[9] G. Charles, R. Goodall, R. Dixon, Model-based condition monitoring at the wheel-rail interface, Vehicle System Dynamics 46 (sup1) (2008) 415-430. doi:10.1080/00423110801979259.

[10] C. Ward, R. Goodall, R. Dixon, Creep Force Estimation at the WheelRail interface, in: Proceedings of the 22nd International Symposium on Dynamics of Vehichles on Roads and Tracks, (C) Manchester Metropolitan University, Machester, 2011.

[11] P. Hubbard, C. Ward, R. Goodall, R. Dixon, Real Time Detection of Low Adhesion in the Wheel/Rail Contact, Proceedings of the Institution of Mechanical Engineers, Part F: Journal of Rail and Rapid Transitdoi:10.1177/0954409713503634. URL http://pif . sagepub . com/content/early/2013/09/17/0954409713503634. abstract

${ }_{410}$ [12] P. Hubbard, G. Amarantidis, C. Ward, Leaves on the Line: Low Adhesion Detection in Railways, in: 7TH IFAC SYMPOSIUM ON MECHATRONIC SYSTEMS \& 15TH MECHATRONICS FORUM INTERNATIONAL CONFERENCE, Loughborough, UK, 2016. 
[13] R. E. Kalman, A New Approach to Linear Filtering and Prediction Problems 182 (Series D) (1960) 35-45.

[14] Dassault, SimPack (2016). URL http://www.3ds.com/products-services/simulia/products/simpack/

[15] R. Goodall, C. Ward, Active control of railway bogies assessment of control strategies in: The International Symposium on Speed-up and Sustainable Technology for Railway and Maglev Systems, Chiba, Japan, 2015, pp. 0-10.

URL https://dspace.lboro.ac.uk/2134/20741

[16] P. D. Hubbard, C. Ward, R. Dixon, R. Goodall, Models for estimation of creep forces in the wheel/rail contact under varying adhesion levels, Vehicle System Dynamics $52 \quad$ (sup1) (2014) 370-386.

425 doi:10.1080/00423114.2014.901541.

URL http://dx.doi.org/10.1080/00423114.2014.901541

[17] J. J. Kalker, On the rolling contact of two elastic bodies in the presence of dry friction, Dissertation, Delft University of Technology (1967).

[18] G. Charles, R. Dixon, R. Goodall, Condition Monitoring Approaches To Estimating Wheel-Rail Profile, in: Proceedings of the UKACC International Conference on Control, Manchester, UK, 2006.

[19] P. Hubbard, C. P. Ward, R. Dixon, R. M. Goodall, Real Time Detection of Low Adhesion in the Wheel / Rail Contact, in: RRUKA Annual Conference, no. November, 2012, pp. 1-5.

[20] T. Söderström, P. Stoica, System identification, Prentice-Hall, Inc., 1988.

[21] L. E. Schwer, Validation metrics for response histories: perspectives and case studies, Engineering with Computers 23 (4) (2007) 295-309.

[22] Mathworks, Matlab Documentation Centre: lqe (2016). URL https://uk.mathworks .com/help/control/ref/kalman.html 